\title{
Group approximation in Cayley topology and coarse geometry, III: Geometric property (T)
}

\author{
MASATO MiMURA \\ NARUTAKA OZAWA \\ HIROKI SAKO \\ YUHEI SUZUKI
}

\begin{abstract}
In this series of papers, we study the correspondence between the following: (1) the large scale structure of the metric space $\bigsqcup_{m}$ Cay $\left(\mathbb{G}^{(m)}\right)$ consisting of Cayley graphs of finite groups with $k$ generators; (2) the structure of groups that appear in the boundary of the set $\left\{\mathbb{G}^{(m)}\right\}$ in the space of $k$-marked groups. In this third part of the series, we show the correspondence among the metric properties "geometric property (T)", “cohomological property (T)" and the group property "Kazhdan's property (T)". Geometric property (T) of Willett-Yu is stronger than being expander graphs. Cohomological property $(\mathrm{T})$ is stronger than geometric property $(\mathrm{T})$ for general coarse spaces.
\end{abstract}

20F65; 46M20

\section{Introduction}

In 1967, D Kazhdan introduced the concept of property (T) for locally compact groups in terms of uniform spectral gaps for all unitary representations (in this paper, we regard Proposition 2.9 as a definition of property (T) for discrete groups), which represents extreme rigidity of groups. See the book of Bekka, de la Harpe and Valette [1] for comprehensive treatise on this property. For instance, G Margulis has observed that for a residually finite and finitely generated group $G$ with property (T), any box space $\square G$ forms a family of expanders, namely, a family of uniformly locally finite and finite connected graphs whose combinatorial Laplacians have the first positive eigenvalues bounded away from zero. (On expanders, we refer the reader to a book [12] by A Lubotzky). Here for such $G$ and a sequence of normal subgroups $N_{1}>N_{2}>\cdots$ of $G$ with finite indices with $\bigcap_{m} N_{m}=\left\{1_{G}\right\}$, the box space $\square_{\left\{N_{m}\right\}_{m}} G$ associated with $\left\{N_{m}\right\}_{m}$ is the coarse disjoint union (see Section 2.3) of finite Cayley graphs $\operatorname{Cay}\left(G / N_{m}, S\right)$, where $S$ is a fixed finite generating set of $G$ (the coarse structure of the box space does not depend on the choice of $S$ ). Expander sequences represent 
strong rigidity, and some expander sequences are known to serve as counterexamples to the surjective side of the coarse Baum-Connes conjecture for coarse spaces. We refer the reader to the monographs Nowak and Yu [18] and Roe [23] for the basics of this subject.

We however may obtain expander sequence from a group far from having property $(\mathrm{T})$. For instance, A Selberg has showed that for a concrete example of $\left\{N_{m}\right\}_{m}$ for $F_{2}$, the free group of rank 2, the box space $\square_{\left\{N_{m}\right\}_{m}} F_{2}$ forms an expander sequence (for details, see a forthcoming book [13] of Lubotzky and Zuk on property $(\tau))$. There has been a lot of interest in the problem of whether one can distinguish expanders coming from property $(\mathrm{T})$ groups from ones coming from non-(T) groups in terms of coarse geometric properties. This problem is related to a question by $\mathrm{J}$ Roe [23] to define "coarse property $(\mathrm{T})$ ".

$\mathrm{R}$ Willett and G Yu $[29 ; 30]$ have studied the maximal coarse Baum-Connes conjecture and introduced the notion of geometric property $(\mathrm{T})$ for a (coarse) disjoint union of uniformly locally finite and finite graphs, which is stronger than being an expander sequence. They have showed that this property is an obstruction to the surjectivity of the maximal coarse Baum-Connes assembly map, and that a box space $\square G$ has geometric property (T) if and only if $G$ possesses property (T). In the later work [31], they have extended the definition of geometric property $(\mathrm{T})$ for (weakly) monogenic coarse spaces of bounded geometry, and proved that this property is a coarse invariant. In this manner, they give a satisfactory answer to the problem mentioned above (on the other hand, the Selberg expander $\square_{\left\{N_{m}\right\}} F_{2}$ is shown in Chen, Wang and Wang [3] to admit a fibered coarse embedding into a Hilbert space in the sense of Chen, Wang and $\mathrm{Yu}$ [4], and this ensures the maximal coarse Baum-Connes conjecture for this space; see also [30]).

It is a well-known theorem of Delorme and Guichardet [1, Theorem 2.12.4] that property $(\mathrm{T})$ can be characterized in terms of 1-cohomology with coefficients in unitary representations. In Section 6, we investigate an analogue of this characterization in the setting of coarse geometry, and introduce cohomological property (T) for coarse spaces. It will be proved that cohomological property $(\mathrm{T})$ implies geometric property (T) (but not vice versa).

The goal of this paper is to provide a characterization of the (coarse) disjoint union $X:=\bigsqcup_{m} \operatorname{Cay}\left(G^{(m)}, s_{1}^{(m)}, s_{2}^{(m)}, \ldots, s_{k}^{(m)}\right)$ of finite Cayley graphs to enjoy geometric property $(\mathrm{T})$. In the previous works of the first-named and the third-named authors, we have revealed that the concept of the space of marked groups and Cayley topology play a key role in studying coarse geometric properties for such $X$. More precisely, 
for the Cayley boundary

$$
\partial_{\text {Cay }}\left(\left\{\mathbb{G}^{(m)}\right\}_{m}\right):={\overline{\left\{\mathbb{G}^{(m)}\right\}_{m}}}^{\text {Cayley }} \backslash\left\{\mathbb{G}^{(m)}\right\}_{m}
$$

of a sequence

$$
\left\{\mathbb{G}^{(m)}=\left(G^{(m)}, s_{1}^{(m)}, s_{2}^{(m)}, \ldots, s_{k}^{(m)}\right)\right\} m
$$

in the space $\mathcal{G}(k)$ of $k$-marked groups, the following holds (for details, we refer the reader to the corresponding papers):

(i) [16]: $X$ as above has property $\mathrm{A} \Leftrightarrow \partial_{\text {Cay }}\left(\left\{\mathbb{G}^{(m)}\right\}_{m}\right)$ is uniformly amenable $\Leftrightarrow$ every member of $\partial_{\text {Cay }}\left(\left\{\mathbb{G}^{(m)}\right\}_{m}\right)$ is amenable.

(ii) [17]: $X$ as above admits a fibered coarse embedding into a Hilbert space $\Leftrightarrow$ $\partial_{\text {Cay }}\left(\left\{\mathbb{G}^{(m)}\right\}_{m}\right)$ is uniformly a-T-menable.

For the definition of $\mathcal{G}(k)$, and the Cayley topology, see Section 2.2. We note that the two results above can be regarded as generalization of previously known results (respectively by E Guentner, and Chen, Wang and Wang [3]) for box spaces. Indeed, in the box space case, for a fixed finite generating set $S=\left(s_{1}, \ldots, s_{k}\right)$ of $G$, the sequence $\left\{\left(G / N_{m}, S\right)\right\}_{m}$ converges to $(G, S)$ in the Cayley topology and hence the singleton $\{(G, S)\}$ is the Cayley boundary of that sequence.

With the notation above, we shall state our main theorem in this paper, which generalizes the above-mentioned result of Willett and Yu for the box spaces.

Theorem 1.1 Let $\left\{\mathbb{G}^{(m)}\right\}_{m \in \mathbb{N}}=\left\{\left(G^{(m)}, s_{1}^{(m)}, s_{2}^{(m)}, \ldots, s_{k}^{(m)}\right)\right\}_{m \in \mathbb{N}}$ be a sequence of finite $k$-marked groups, and let $X=\bigsqcup_{m \in \mathbb{N}} \operatorname{Cay}\left(\mathbb{G}^{(m)}\right)$ be their disjoint union. Then the following are equivalent.

(1) Every member of $\partial_{\text {Cay }}\left(\left\{\mathbb{G}^{(m)}\right\}_{m}\right)$ has property (T) of Kazhdan.

(2) The metric space $X$ has geometric property (T).

(3) The metric space $X$ has cohomological property (T).

Theorem 1.1 provides us with the following corollary, which generalizes the result of Margulis mentioned above:

Corollary 1.2 Let $\left\{\mathbb{G}^{(m)}\right\}_{m \in \mathbb{N}}=\left\{\left(G^{(m)}, s_{0}^{(m)}, s_{2}^{(m)}, \ldots, s_{k}^{(m)}\right)\right\}_{m \in \mathbb{N}}$ be a sequence of finite $k$-marked groups. If every member of $\partial_{\text {Cay }}\left(\left\{\mathbb{G}^{(m)}\right\}_{m}\right)$ has property $(\mathrm{T})$, then the sequence of Cayley graphs $\left\{\mathrm{Cay}\left(\mathbb{G}^{(m)}\right)\right\}_{m}$ forms an expander family. 
In fact, the proof of Corollary 1.2 does not require the notion of geometric property $(\mathrm{T})$ and follows directly from the intermediate result, Proposition 5.1. See also [15] for a quantitative version of Corollary 1.2. The proof of Theorem 1.1 is scattered in this paper: $(2) \Rightarrow(1)$ is proved in Section 3, the converse in Section 4, and (2) $\Leftrightarrow$ (3) in Section 6. In these proofs, we have avoided the technical aspects of $\mathrm{C}^{*}$-algebra theory. Instead, they are organized at the end of this paper (Section 7), where we study the structure of the maximal uniform Roe algebra in detail.

Acknowledgments This work gained momentum when the authors attended the conference "Metric Geometry and Analysis" held at Kyoto University in December 2013, and was finished during the first and second-named authors were visiting Institut Henri Poincaré in the spring 2014 for the trimester on "Random Walks and Asymptotic Geometry of Groups". We gratefully acknowledge their hospitality and stimulating environments. The authors benefited from various comments and suggestions by Romain Tessera and Rufus Willett. The authors would like to thank Shin-ichi Oguni for providing us with literature and information on (co)homology theory in coarse geometry. The first-named author was supported by JSPS Grant-in-Aid for Young Scientists (B) number 25800033. The second-named author was supported by JSPS KAKENHI Grant number 23540233. The fourth-named author was supported by JSPS Research Fellow number 25-7810, and the MEXT Program of Leading Graduate Schools.

\section{Preliminaries}

\subsection{Coarse equivalence}

Recall from [23, Definition 1.8] that a map $f: X \rightarrow Y$ between metric spaces is said to be uniformly bornologous if $\sup \{d(f(x), f(y)) \mid d(x, y) \leq R\}<\infty$ for every $R>0$. Two maps $f_{i}: X \rightarrow Y$ are close if $\sup _{x} d\left(f_{1}(x), f_{2}(x)\right)<\infty$. The two metric spaces $X$ and $Y$ are coarsely equivalent if there are uniformly bornologous maps $f: X \rightarrow Y$ and $g: Y \rightarrow X$ such that $g \circ f$ and $f \circ g$ are close to $\operatorname{id}_{X}$ and $\operatorname{id}_{Y}$, respectively. Thus, for every $n \in \mathbb{N}$, the $n$-point metric space $n=\{1, \ldots, n\}$ (say $d(i, j)=|i-j|$ ) is coarsely equivalent to a point, and the metric spaces $Y$ and $Y \times \boldsymbol{n}$ (say, $d((y, i),(z, j))=d(y, z)+|i-j|$ ) are coarsely equivalent, via the inclusion $\iota: Y \hookrightarrow Y \times\{1\}$ and the projection pr: $Y \times \boldsymbol{n} \rightarrow Y$. Every coarse equivalence roughly arises in this way.

Lemma 2.1 If $f: X \rightarrow Y$ is a coarse equivalence between uniformly locally finite

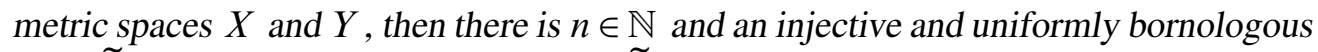
map $\tilde{f}: X \rightarrow Y \times \boldsymbol{n}$ such that $f=\operatorname{pr} \circ \tilde{f}$. 
Proof Since the $\left\{f^{-1}(y)\right\} y$ are uniformly bounded subsets of a uniformly locally finite metric space $X$, a matching theorem yields a finite partition $X=\bigsqcup_{i=1}^{n} X_{i}$ such that $f$ is injective on each of the $X_{i}$. If we define $\tilde{f}(x)=(f(x), i)$ for $x \in X_{i}$, then we are done.

\subsection{Cayley topology}

The space $\mathcal{G}(k)$ of $k$-marked groups and the Cayley topology on it enable us to regard a finitely generated group as a point in a topological space.

Let $\mathbb{G}=\left(G, s_{1}, s_{2}, \ldots, s_{k}\right)$ be a $(k+1)$-tuple of a group $G$ and its generators $s_{1}, \ldots s_{k}$. We call such an object a $k$-marked group. Throughout this paper, we use the following terminologies:

- A $k$-marked group $\left(H, s_{1}^{\prime}, s_{2}^{\prime}, \ldots, s_{k}^{\prime}\right)$ is called a quotient of $\left(G, s_{1}, s_{2}, \ldots, s_{k}\right)$ if there exists a group homomorphism $\phi: G \rightarrow H$ satisfying that $\phi\left(s_{j}\right)=$ $s_{j}^{\prime}$ for all $j=1, \ldots, k$. Note that every member of $\mathcal{G}(k)$ is a quotient of $\left(F_{k}, a_{1}, a_{2}, \ldots, a_{k}\right)$, where $F_{k}$ is the free group generated by $a_{1}, \ldots, a_{k}$.

- If the above homomorphism $\phi$ is isomorphic, then two $k$-marked groups $\left(G, s_{1}, s_{2}, \ldots, s_{k}\right)$ and $\left(H, s_{1}^{\prime}, s_{2}^{\prime}, \ldots, s_{k}^{\prime}\right)$ are said to be isomorphic.

- A $k$-marked group $\left(G, s_{1}, s_{2}, \ldots, s_{k}\right)$ is said to be finitely presented if there exists a finite subset $A \subset F_{k}$ such that the minimal normal subgroup $N \subset F_{k}$ containing $A$ is the kernel of the quotient map $\phi:\left(F_{k}, a_{1}, \ldots, a_{k}\right) \rightarrow\left(G, s_{1}, \ldots, s_{k}\right)$.

We note that the finite presentability of a group is independent of the choices of markings (see [10, V.2]). We denote by $\mathcal{G}(k)$ the set of the isomorphism classes of $k$-marked groups and call it the space of $k$-marked groups or the Cayley topological space. A natural topology on $\mathcal{G}(k)$ was introduced by Grigorchuk in [7]. We call it the Cayley topology. For details, see the book [10, Section V.10] of de la Harpe. We also recall the notation used in the paper that is part I of this series, [16] by the first-named and the third-named authors. The topology is generated by relations. More precisely, it is generated by closed and open subsets

$$
O\left(s_{j(1)}^{\epsilon(1)} s_{j(2)}^{\epsilon(2)} \cdots s_{j(n)}^{\epsilon(n)}\right)=\left\{\left(G, s_{1}, s_{2}, \ldots, s_{k}\right) \in \mathcal{G}(k) \mid s_{j(1)}^{\epsilon(1)} s_{j(2)}^{\epsilon(2)} \cdots s_{j(n)}^{\epsilon(n)}=1_{G}\right\} .
$$

Here $s_{j(l)}$ is one of the generators $\left\{s_{1}, s_{2}, \ldots, s_{k}\right\}$ and $\epsilon(l)$ is either 1 or -1 . The resulting topology is known to be Hausdorff (in fact metrizable) and compact. Two $k$-marked groups are close if the balls with large radius of their Cayley graphs are identical. We may regard the following as a definition. 
Lemma 2.2 [16, Lemma 2.6] Let $\mathbb{G}=\left(G, s_{1}, \ldots, s_{k}\right)$ be a $k$-marked group and $R$ be a constant greater than 1 . Let $N(\mathbb{G}, R)$ be the subset of $\mathcal{G}(k)$ consisting of $k$-marked groups $\mathbb{H}=\left(H, s_{1}^{\prime}, \ldots, s_{k}^{\prime}\right)$ satisfying that there exists a bijection $\phi: B\left(1_{H}, 2 R\right) \rightarrow B\left(1_{G}, 2 R\right)$ from the ball of $\mathbb{H}$ to that of $\mathbb{G}$ with radius $2 R$ such that

- $\phi\left(s_{j}^{\prime}\right)=s_{j}$ for every $j=1, \ldots, k$,

- $\phi\left(g^{-1}\right)=\phi(g)^{-1}$ for every $g \in B\left(1_{H}, 2 R\right)$,

- $\phi(g h)=\phi(g) \phi(h)$ for every $g, h \in B\left(1_{H}, R\right)$.

The subsets $\{N(\mathbb{G}, R)\}_{R}$ form a neighborhood system of $\mathbb{G} \in \mathcal{G}(k)$.

We call any $\phi$ satisfying the three conditions in the lemma a partial isomorphism.

Lemma 2.3 Let $\mathbb{P}=\left(P, \sigma_{1}, \ldots, \sigma_{k}\right) \in \mathcal{G}(k)$ be a $k$-marked group. Assume that $P$ is finitely presented. The set of all the quotient groups of $\mathbb{P}$

$$
\mathcal{Q}_{\mathbb{P}}=\left\{\begin{array}{l|l}
\left(G, s_{1}, \ldots, s_{k}\right) \in \mathcal{G}(k) \mid \begin{array}{l}
\text { there exists a surjective homomorphism } \\
\phi: P \rightarrow G \text { such that } \phi\left(\sigma_{j}\right)=s_{j}
\end{array}
\end{array}\right\}
$$

is a closed and open subset of $\mathcal{G}(k)$.

Proof For each relation $s_{j(1)}^{\epsilon(1)} s_{j(2)}^{\epsilon(2)} \cdots s_{j(n)}^{\epsilon(n)}$ defining $\mathbb{P}$, the subset

$$
O\left(s_{j(1)}^{\epsilon(1)} s_{j(2)}^{\epsilon(2)} \cdots s_{j(n)}^{\epsilon(n)}\right) \subset \mathcal{G}(k)
$$

is open and closed. So is the finite intersection of such subsets corresponding to the relations in $\mathbb{P}$. The intersection is nothing other than $\mathcal{Q}_{\mathbb{P}}$.

Finally, we record the following fact. For the proof, take $\mathbb{P}_{R}$ to be the marked group quotient of $\mathbb{F}_{k}$ ( $F_{k}$ with the standard generators) by all the relations of $\mathbb{G}$ that have length at most $R$. Note that when $G$ is finitely presented, $\mathbb{P}_{R}$ eventually coincides with $\mathbb{G}$.

Lemma 2.4 For every $\mathbb{G}=\left(G, s_{1}, \ldots, s_{k}\right) \in \mathcal{G}(k)$, there exists a sequence of finitely presented groups $\left\{\mathbb{P}_{R}=\left(P_{R}, s_{1}^{(R)}, \ldots, s_{k}^{(R)}\right)\right\}_{R \in \mathbb{N}}$ converging to $\mathbb{G}$ such that

- $\mathbb{G}$ is a quotient of $\mathbb{P}_{R}$ for every $R$,

- $\mathbb{P}_{R+1}$ is a quotient of $\mathbb{P}_{R}$ for every $R$. 


\subsection{Marked groups as metric spaces}

Let $(X, d)$ be a metric space (for which we allow $d$ to take the value $\infty$ ). The space $X$ is said to be uniformly locally finite (or to have bounded geometry) if $\sup _{x \in X} \sharp(B(x, R))<\infty$ for every $R>0$. Throughout this paper, $B(x, R)$ denotes the closed ball with radius $R$ whose center is $x$.

Marked groups have provided interesting examples in metric geometry. For a $k$-marked group $\mathbb{G}=\left(G, s_{1}, \ldots, s_{k}\right)$, the word metric $d: G \times G \rightarrow[0, \infty)$ is defined as

$$
d(g, h)=\min \left\{n \mid \begin{array}{c}
\exists n, \exists j(1), \ldots, j(n), \exists \epsilon(1), \ldots, \epsilon(n) \in\{1,-1\}, \\
g h^{-1}=s_{j(1)}^{\epsilon(1)} s_{j(2)}^{\epsilon(2)} \cdots s_{j(n)}^{\epsilon(n)}
\end{array}\right\}
$$

(or zero if $g=h$ ). Note that we are using the right-invariant word metric. In this way, the group $G$ becomes a uniformly locally finite metric space. (It is the vertex set of the Cayley graph equipped with the edge metric.) We denote this metric space by $\operatorname{Cay}(\mathbb{G})=\operatorname{Cay}\left(G, s_{1}, \ldots, s_{k}\right)$. The coarse equivalence class of the metric on $G$ is independent of the choice of generating subset $\left\{s_{1}, \ldots, s_{k}\right\}$.

The subject of this paper is a metric space of the form

$$
X=\bigsqcup_{m \in \mathbb{N}} \operatorname{Cay}\left(G^{(m)}, s_{1}^{(m)}, \ldots, s_{k}^{(m)}\right) .
$$

where $\left\{\left(G^{(m)}, s_{1}^{(m)}, \ldots, s_{k}^{(m)}\right)\right\}_{m}$ is a sequence of finite $k$-marked groups. Note that the coarse structure of $X$ does depend on the choice of a family $\left\{s_{1}^{(m)}, \ldots, s_{k}^{(m)}\right\}_{m}$ of generating subsets. For a disjoint union $X=\bigsqcup_{m} X^{(m)}$ of metric spaces, it is customary in coarse geometry to put a metric $d$ on $X$ in such a way that it coincides with the original metric on each of the $X^{(m)}$ and that $d\left(X^{(m)}, X^{(n)}\right) \rightarrow \infty$ as $|m-n|(m+n) \rightarrow \infty$. Such a metric is unique up to coarse equivalence. We call this metric space the coarse disjoint union of $\left\{X^{(m)}\right\}_{m}$ and denote it by $\bigsqcup_{m} X^{(m)}$. However, it is more convenient to allow our metric $d$ to take the value $\infty$, and define the distance between two points of distinct components to be $\infty$. We call this (generalized) metric space the disjoint union of $\left\{X^{(m)}\right\}_{m}$ and denote it simply by $\bigsqcup_{m} X^{(m)}$, as we deal with it most of the time throughout this paper. These two notions of a disjoint union do not make much difference and the precise relation between these will be described in the end of Section 7.

\subsection{Algebraic uniform Roe algebra and group algebra}

For a uniformly locally finite metric space $(X, d)$, the algebraic uniform Roe algebra $\mathbb{C}_{\mathrm{u}}[X]$ is defined to be the collection of all matrices indexed by $X$ whose propagation 
is finite. More precisely,

$$
\mathbb{C}_{\mathrm{u}}[X]=\left\{\left[a_{x, y}\right]_{x, y \in X}\left|\sup _{x, y}\right| a_{x, y} \mid<\infty \text { and } \operatorname{prop}(a)<\infty\right\},
$$

where $\operatorname{prop}(a)=\sup \left\{d(x, y) \mid a_{x, y} \neq 0\right\}$ is the propagation of a matrix $a=\left[a_{x, y}\right]_{x, y \in X}$. The usual multiplication between two matrices defines the product on $\mathbb{C}_{\mathrm{u}}[X]$. The conjugate transpose $a \mapsto a^{*}=\left[\overline{a_{y, x}}\right]_{x, y \in X}$ defines the involution $*$ on the algebra. The diagonal algebra of $\mathbb{C}_{\mathrm{u}}[X]$ is canonically isomorphic to the algebra $\ell_{\infty}(X)$ of bounded functions and hence simply denoted by $\ell_{\infty}(X)$.

Recall that $t=\left[t_{x, y}\right]_{x, y} \in \mathbb{C}_{\mathrm{u}}[X]$ is called a partial translation if there is a bijection $\phi_{t}$ from a subset $A=\operatorname{dom}\left(\phi_{t}\right) \subset X$ onto a subset $B=\operatorname{ran}\left(\phi_{t}\right) \subset X$ such that $t_{x, y}=1$ if $y \in A$ and $x=\phi_{t}(y)$; or else $t_{x, y}=0$. We will identify the partial translation $t$ with the partially defined bijection $\phi_{t}$. A partial translation that is a bijection on $X$ is called a full translation. We record the following well-known fact as a lemma.

Lemma 2.5 If Cay $(G)$ is the Cayley metric space of a finitely generated group $G$, then $\mathbb{C}_{\mathrm{u}}[\mathrm{Cay}(G)]$ is isomorphic to the algebraic crossed product $\ell_{\infty}(G) \rtimes G$. In general, if $X$ is a uniformly locally finite metric space and $\Gamma_{X}$ denotes the group of full translations in $\mathbb{C}_{\mathrm{u}}[X]$, then $\mathbb{C}_{\mathrm{u}}[X]=\operatorname{span}\left(\ell_{\infty}(X) \Gamma_{X}\right)$.

Proof We sketch the proof of the second assertion for the reader's convenience. It suffices to show that every partial translation $t$ belongs to the latter set. For the bijection $t: A \rightarrow B$ as above, there is a partition $A=\bigsqcup_{i=0}^{3} A_{i}$ such that $\left.t\right|_{A_{0}}=\mathrm{id}_{A_{0}}$ and $t\left(A_{i}\right) \cap A_{i}=\varnothing$ for $i=1,2,3$ (take a maximal $A_{1}$ as such). Then, $\left(\left.t\right|_{A_{i}}\right) \sqcup\left(\left.t^{-1}\right|_{t\left(A_{i}\right)}\right)$ extends to a full translation by setting it equal to the identity outside of $A_{i} \sqcup t\left(A_{i}\right)$.

The maximal $\mathrm{C}^{*}$-norm on $\mathbb{C}_{\mathrm{u}}[X]$ is defined as

$$
\|a\|_{\max }=\sup \left\{\|\pi(a)\| \mid \pi: \mathbb{C}_{\mathrm{u}}[X] \rightarrow \mathbb{B}(\mathcal{H}) * \text {-representation on a Hilbert space }\right\} .
$$

Denote by $\mathrm{C}_{\mathrm{u}, \max }^{*}[X]$ the completion of $\mathbb{C}_{\mathrm{u}}[X]$ with respect to $\|\cdot\|_{\max }$, and call it the maximal uniform Roe algebra. Note that when $X$ has property A, the norm $\|\cdot\|_{\max }$ coincides with the norm $\|\cdot\|_{\mathbb{B}\left(\ell_{2}(X)\right)}$ as an operator on $\ell_{2}(X)$ (see [26, Proposition 1.3]).

For a group $G$, we denote by $\mathrm{C}_{\max }^{*}[G]$ the completion of the group algebra $\mathbb{C}[G]$ with respect to the maximal $\mathrm{C}^{*}$-norm $\|\cdot\|_{\max }$. This $\mathrm{C}^{*}$-algebra is called the maximal (or full) group $\mathrm{C}^{*}$-algebra. We have an natural embedding of the group algebra $\mathbb{C}[G]$ into $\mathbb{C}_{\mathrm{u}}[\operatorname{Cay}(G)]$, given by $\xi \mapsto\left[\xi\left(g h^{-1}\right)\right]_{g, h}$, but the corresponding homomorphism $\mathrm{C}_{\max }^{*}[G] \rightarrow \mathrm{C}_{\mathrm{u}, \max }^{*}[X]$ is not faithful unless $G$ is amenable. 


\subsection{Sum of squares in $*$-algebras}

For a $*$-algebra, a notion of positivity is defined as follows.

Definition 2.6 For a $*$-algebra $\mathcal{A}$, the cone of sums of squares is defined to be

$$
\Sigma^{2} \mathcal{A}=\left\{\sum_{i=1}^{n} \xi_{i}^{*} \xi_{i} \mid n \in \mathbb{N}, \xi_{1}, \ldots, \xi_{n} \in \mathcal{A}\right\} .
$$

In case $\mathcal{A}=\mathbb{C}_{\mathrm{u}}[\operatorname{Cay}(G)]$ or $\mathcal{A}=\mathbb{C}[G]$, we say an element $x \in \mathcal{A}$ is a sum of squares of $n$ elements with propagation at most $R$ if there are $n$ elements $\left\{\xi_{i}\right\}_{i=1}^{n}$ in $\mathcal{A}$ such that $\operatorname{prop}\left(\xi_{i}\right) \leq R$ and $x=\sum_{i=1}^{n} \xi_{i}^{*} \xi_{i}$. We denote by $\Sigma_{n, R}^{2} \mathcal{A}$ the set of sums of squares of $n$ elements with propagation at most $R$.

Lemma 2.7 Let $\mathbb{G}$ be an amenable $k$-marked group. Let $x$ be an element in the group algebra $\mathbb{C}[G]$. Suppose that $x=\sum_{i=1}^{n} \xi_{i}^{*} \xi_{i}$ is a sum of squares of $n$ elements $\left\{\xi_{i}\right\}_{i=1}^{n}$ in $\mathbb{C}_{\mathrm{u}}[\mathrm{Cay}(\mathbb{G})]$ with propagation at most $R$. Then $x$ is also a sum of squares as an element of $\mathbb{C}[G]$. More precisely, $x$ is a sum of squares of $\left(n \times \sharp\left(B\left(1_{G}, R\right)\right)\right)$ elements in $\mathbb{C}[G]$ with propagation at most $R$.

Proof Fix a mean $\Psi$ on $\ell_{\infty}(G)$ that is invariant under the left translation action $\left\{l_{g}: \ell_{\infty}(G) \rightarrow \ell_{\infty}(G)\right\}_{g \in G}$. Note that every element $x$ in $\mathbb{C}_{\mathrm{u}}[\operatorname{Cay}(\mathbb{G})]$ is uniquely written as $x=\sum_{g} \xi_{g} g$, where $\xi_{g} \in \ell_{\infty}(G) \subset \mathbb{C}_{\mathrm{u}}[\operatorname{Cay}(\mathbb{G})]$ (all but finitely many are zero) and $g \in G \subset \mathbb{C}[G]$. We extend the mean $\Psi$ to $\widetilde{\Psi}: \mathbb{C}_{\mathrm{u}}[\mathrm{Cay}(\mathbb{G})] \rightarrow \mathbb{C}[G]$ by $\widetilde{\Psi}\left(\sum_{g} \xi_{g} g\right)=\sum_{g} \Psi\left(\xi_{g}\right) g$. Since $x=\sum_{i=1}^{n} \xi_{i}^{*} \xi_{i} \in \mathbb{C}[G] \cap \Sigma^{2} \mathbb{C}_{\mathrm{u}}[\operatorname{Cay}(\mathbb{G})]$ implies $x=\sum_{i=1}^{n} \widetilde{\Psi}\left(\xi_{i}^{*} \xi_{i}\right)$, it suffices to show $\widetilde{\Psi}\left(\xi^{*} \xi\right) \in \Sigma_{N, R}^{2} \mathbb{C}[G]$ for every $\xi \in \mathbb{C}_{\mathrm{u}}[\operatorname{Cay}(\mathbb{G})]$ such that $\operatorname{prop}(\xi) \leq R$. Here $N=\sharp\left(B\left(1_{G}, R\right)\right)$.

Let $\xi=\sum_{g \in B\left(1_{G}, R\right)} \xi_{g} g$ be given. Then,

$$
\widetilde{\Psi}\left(\xi^{*} \xi\right)=\sum_{g, h} \tilde{\Psi}\left(l_{h^{-1}}\left(\overline{\xi_{h}} \xi_{g}\right) h^{-1} g\right)=\sum_{g, h} \Psi\left(\overline{\xi_{h}} \xi_{g}\right) h^{-1} g .
$$

Since $\Psi: \ell_{\infty}(G) \rightarrow \mathbb{C}$ is a positive linear functional, the matrix $\left[\Psi\left(\overline{\xi_{h}} \xi_{g}\right)\right]_{g, h \in B\left(1_{G}, R\right)}$ is positive semi-definite. Considering the square root of the matrix, we obtain vectors $\left\{\alpha_{g}\right\}_{g \in B\left(1_{G}, R\right)} \subset \mathbb{C}^{N}$ satisfying that $\Psi\left(\bar{\xi}_{h} \xi_{g}\right)=\left\langle\alpha_{g}, \alpha_{h}\right\rangle$. Write $\alpha_{g}=\left(\alpha_{g}^{(i)}\right)_{i=1}^{N} \in \mathbb{C}^{N}$. Then, one sees

$$
\widetilde{\Psi}\left(\xi^{*} \xi\right)=\sum_{g, h}\left\langle\alpha_{g}, \alpha_{h}\right\rangle h^{-1} g=\sum_{i=1}^{N}\left(\sum_{h} \alpha_{h}^{(i)} h\right)^{*}\left(\sum_{g} \alpha_{g}^{(i)} g\right)
$$

and so $\tilde{\Psi}\left(\xi^{*} \xi\right) \in \Sigma_{N, R}^{2} \mathbb{C}[G]$. This completes the proof. (In effect, we have shown that $\widetilde{\Psi}$ is completely positive in the sense of [21, Section 12].) 


\subsection{Positive elements in maximal algebras}

As a simple application of theory of semi-pre- $C^{*}$-algebras, we obtain the following. For details, see [24, Proposition 15] or [21, Theorem 1].

Proposition 2.8 Let $\mathcal{A}$ be either the group algebra $\mathbb{C}[G]$ of a group $G$ or the algebraic uniform Roe algebra $\mathbb{C}_{\mathrm{u}}[X]$ of a uniformly locally finite metric space $X$. Let $a \in \mathcal{A}$ be a self-adjoint element. Then the element $a$ is positive in respectively $\mathrm{C}_{\max }^{*}[G]$ or $\mathrm{C}_{\mathrm{u}, \max }^{*}[X]$ if and only if $a+\epsilon 1 \in \Sigma^{2} \mathcal{A}$ for every $\epsilon>0$.

\subsection{On property (T)}

For a $k$-marked group $\left(G, s_{1}, \ldots, s_{k}\right)$, let $\Delta$ denote the (nonnormalized) Laplacian

$$
2 k-\sum_{j=1}^{k}\left(s_{j}+s_{j}^{-1}\right) \in \mathbb{C}[G] .
$$

Property (T) of Kazhdan can be formulated as follows.

Proposition 2.9 (See [2, Lemma 12.1.8]) The following are equivalent:

- The group $G$ has property (T).

- The spectrum of $\Delta$ in the $\mathrm{C}^{*}$-algebra $\mathrm{C}_{\max }^{*}[G]$ has a gap. More precisely, there exists $v>0$ such that the spectrum of $\Delta$ is included in $\{0\} \cup[v, \infty)$.

\subsection{Geometric property $(T)$}

Let $\left\{X^{(m)}\right\}_{m}$ be a sequence of finite connected graphs whose degree is uniformly bounded. A notion called geometric property $(\mathrm{T})$ is defined for the disjoint union $X=\bigsqcup_{m=1}^{\infty} X^{(m)}$, or more generally for (weakly) monogenic coarse spaces having bounded geometry in [31]. If $X$ has geometric property (T) and the cardinality $\sharp\left(X^{(m)}\right)$ of components tends to $\infty$, then it is a sequence of expander graphs. The converse need not hold; see the introduction. Geometric property $(\mathrm{T})$ was originally introduced by Willett and Yu in their study of the maximal coarse Baum-Connes conjecture [30].

In this paper, we take the second condition in the following proposition as a definition of geometric property $(\mathrm{T})$. Let $\Delta_{m}$ be the (nonnormalized) discrete Laplacian on $\ell_{2}\left(X^{(m)}\right)$ :

$$
\left(\Delta_{m}\right)_{x, y}:= \begin{cases}-1 & \text { if } d(x, y)=1 \\ \operatorname{deg}(x) & \text { if } x=y \\ 0 & \text { otherwise. }\end{cases}
$$


The sequence $\Delta=\left(\Delta_{m}\right)_{m \in \mathbb{N}}$ is an element of $\mathbb{C}_{\mathrm{u}}[X]$ whose propagation is 1 . It is called Laplacian. The Laplacian $\Delta \in \mathbb{C}_{\mathrm{u}}[X]$ is also defined for a general uniformly locally finite metric space $X$. See [31, Section 5].

Proposition 2.10 (See [31, Proposition 5.7]) The following are equivalent:

(1) The space $X$ has geometric property (T).

(2) The Laplacian $\Delta$ in the maximal uniform Roe algebra $\mathrm{C}_{\mathrm{u}, \max }^{*}[X]$ has a spectral gap. More precisely, there exists a positive number $v$ such that the spectrum of $\Delta$ in $\mathrm{C}_{\mathrm{u}, \max }^{*}[X]$ is included in $\{0\} \cup[v, \infty)$.

In this paper, we will study when the disjoint union $X=\bigsqcup_{m \in \mathbb{N}} X^{(m)}$ of a sequence $\left\{X^{(m)}\right\}$ of (finite Cayley) metric spaces has geometric property $(\mathrm{T})$. The answer is quite simple when $X$ is a finite disjoint union.

Corollary 2.11 The disjoint union $X=\bigsqcup_{i=1}^{n} X^{(i)}$ of finitely many spaces $\left\{X_{i}\right\}_{i=1}^{n}$ has geometric property $(\mathrm{T})$ if and only if each $X^{(i)}$ has geometric property $(\mathrm{T})$.

Proof Let $\Delta_{i}$ denote the Laplacian for $X^{(i)}$. It is not difficult to see that $\Delta=\left(\Delta_{i}\right)_{i=1}^{n}$ under the canonical isomorphism $\mathrm{C}_{\mathrm{u}, \max }^{*}[X]=\bigoplus_{i=1}^{n} \mathrm{C}_{\mathrm{u}, \max }^{*}\left[X^{(i)}\right]$.

\section{From geometric property (T) to property (T)}

Let us start the proof of Theorem 1.1: (2) $\Rightarrow(1)$; assuming that the disjoint union $\bigsqcup_{m \in \mathbb{N}} \operatorname{Cay}\left(G^{(m)}, s_{1}^{(m)}, \ldots, s_{k}^{(m)}\right)$ has geometric property $(\mathrm{T})$, we shall prove that every

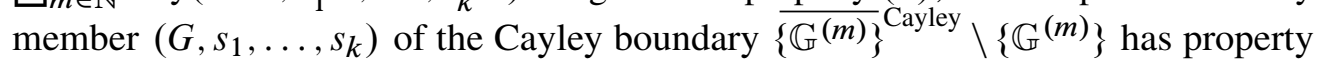
(T) of Kazhdan. By replacing it with a subsequence, we may assume that the sequence $G^{(m)}$ converges to $G$ with respect to the Cayley topology. See also Corollary 2.11.

Let $\Delta_{m}$ denote the discrete (nonnormalized) Laplacian in the group algebra $\mathbb{C}\left[G^{(m)}\right]$, which is given by

$$
\Delta_{m}=2 k-\sum_{j=1}^{k}\left(s_{j}^{(m)}+s_{j}^{(m)^{-1}}\right) .
$$

We view $\Delta_{m}$ also as an element in $\mathbb{C}_{\mathrm{u}}\left[\mathrm{Cay}\left(\mathbb{G}^{(m)}\right)\right]$ and consider the direct product $\Delta=\left(\Delta_{m}\right)_{m} \in \mathbb{C}_{\mathrm{u}}\left[\bigsqcup_{m} \operatorname{Cay}\left(\mathbb{G}^{(m)}\right)\right]$. By assumption, the spectrum of $\Delta$ in the maximal uniform Roe algebra $\mathrm{C}_{\mathrm{u}, \max }^{*}\left[\bigsqcup_{m} \mathrm{Cay}\left(\mathbb{G}^{(m)}\right)\right]$ is included in $\{0\} \cup[v, \infty)$ for some $v>0$ (Proposition 2.10). Thus the element $\Delta^{2}-v \Delta \in \mathbb{C}_{\mathrm{u}}\left[\bigsqcup_{m}\right.$ Cay $\left.\left(\mathbb{G}^{(m)}\right)\right]$ is positive in the $\mathrm{C}^{*}$-algebra $\mathrm{C}_{\mathrm{u} \text {,max }}^{*}\left[\bigsqcup_{m} \operatorname{Cay}\left(\mathbb{G}^{(m)}\right)\right]$, by the spectral mapping theorem. By 
Proposition 2.8, for every positive number $\epsilon$, one has $\Delta^{2}-\nu \Delta+\epsilon=\sum_{i=1}^{n} \eta_{i}^{*} \eta_{i} \epsilon$ $\Sigma^{2} \mathbb{C}_{\mathrm{u}}\left[\bigsqcup_{m} \operatorname{Cay}\left(\mathbb{G}^{(m)}\right)\right]$. Let $R=\max _{i} \operatorname{prop}\left(\eta_{i}\right)$ and choose a large natural number $m$ such that there exists a partial isomorphism $\phi: B\left(1_{G^{(m)}}, 2 R\right) \rightarrow B\left(1_{G}, 2 R\right)$ between balls of $G^{(m)}$ and $G$. Here, $\phi$ satisfies conditions in Lemma 2.2. Taking the $m^{\text {th }}$ entries of $\Delta$ and $\eta_{i}$, we see that $\Delta_{m}{ }^{2}-v \Delta_{m}+\epsilon \in \Sigma_{n, R}^{2} \mathbb{C}_{\mathrm{u}}\left[\operatorname{Cay}\left(\mathbb{G}^{(m)}\right)\right]$. By Lemma 2.7, $\Delta_{m}{ }^{2}-v \Delta_{m}+\epsilon \in \mathbb{C}\left[G^{(m)}\right]$ is a sum of squares of operators $\xi_{i}$ in the group algebra $\mathbb{C}\left[G^{(m)}\right]$ with propagation at most $R$. Extending the partial isomorphism $\phi$ to the linear map $\phi: \mathbb{C}\left[B\left(1_{G^{(m)}}, 2 R\right)\right] \rightarrow \mathbb{C}\left[B\left(1_{G}, 2 R\right)\right]$ and applying it to

$$
\Delta_{m}^{2}-v \Delta_{m}+\epsilon=\sum_{i} \xi_{i}^{*} \xi_{i}
$$

we see that the Laplacian $\Delta_{\mathbb{G}}$ in the group algebra $\mathbb{C}[G]$ satisfies

$$
\Delta_{\mathbb{G}}^{2}-v \Delta_{\mathbb{G}}+\epsilon=\sum_{i} \phi\left(\xi_{i}\right)^{*} \phi\left(\xi_{i}\right) .
$$

This implies that $\Delta_{\mathbb{G}}{ }^{2}-v \Delta_{\mathbb{G}}+\epsilon$ is positive in the maximal group algebra $C_{\max }^{*}[G]$. Since $\epsilon>0$ was arbitrary, the spectrum of $\Delta_{\mathbb{G}}$ is included in $\{0\} \cup[v, \infty)$, by the spectral mapping theorem. This means that $G$ has property (T).

The key step in the above proof is to show that $\Delta_{\mathbb{G}}^{2}-v \Delta_{\mathbb{G}}+\epsilon$ is a sum of squares for every $\epsilon>0$. To check whether a finitely generated group $G$ has property (T), we in fact do not need the extra $\epsilon$.

Theorem 3.1 [20] A marked group $\mathbb{G}$ has property (T) if and only if there exists a positive number $v$ and $\xi_{1}, \ldots, \xi_{n} \in \mathbb{R}[G]$ such that

$$
\Delta_{\mathbb{G}}^{2}-v \Delta_{\mathbb{G}}=\sum_{i=1}^{n} \xi_{i}^{*} \xi_{i} .
$$

\section{From property (T) to geometric property (T)}

Shalom [25, Theorem 6.7] has showed that every property (T) group is a quotient of finitely presented property $(\mathrm{T})$ group, and more generally that property $(\mathrm{T})$ is an open property in the space of marked groups, namely, the set of all $k$-marked property (T) groups is open in $\mathcal{G}(k)$. (This fact also follows from Theorem 3.1 above.) See a survey of Y Stalder [27] for a more general result. Recall that $\mathcal{Q}_{\mathbb{P}}$ stands for the set of marked group quotients of $\mathbb{P}$.

Proposition 4.1 Every $k$-marked group $\mathbb{G}$ with property $(\mathrm{T})$ is a quotient of a finitely presented $k$-marked group $\mathbb{P}$ with property $(\mathrm{T})$. Moreover, for every sequence $\left\{\mathbb{G}^{(m)}\right\}_{m}$ in $\mathcal{G}(k)$ that converges to $\mathbb{G}$ and for every such finitely presented $\mathbb{P}$, there exists $N \in \mathbb{N}$ such that $\left\{\mathbb{G}^{(m)}: m \geq N\right\} \subset \mathcal{Q}_{\mathbb{P}}$. 
Proof The first assertion is proved in [25] and the second follows from Lemma 2.3. See also Lemma 2.4.

Lemma 4.2 Let $K$ be a nonempty closed subset of $\mathcal{G}(k)$. If every element of $K$ has property $(\mathrm{T})$, then there exist finitely many finitely presented property $(\mathrm{T})$ marked groups $\mathbb{P}_{1}, \ldots, \mathbb{P}_{n} \in \mathcal{G}(k)$ such that $K \subset \bigcup_{i} \mathcal{Q}_{\mathbb{P}_{i}}$.

Proof By Proposition 4.1, $\left\{\mathcal{Q}_{\mathbb{P}} \mid P\right.$ is a finitely presented group with property $\left.(\mathrm{T})\right\}$ is an open covering of $K$. Since the subset $K$ is compact, there exist finitely many groups $\mathbb{P}_{1}, \ldots, \mathbb{P}_{n}$ such that $K \subset \bigcup_{i} \mathcal{Q}_{\mathbb{P}_{i}}$.

We begin the proof of Theorem 1.1: (1) $\Rightarrow(2)$. Suppose that every $k$-marked group $\mathbb{G}$ in the boundary

$$
{\overline{\left\{\mathbb{G}^{(m)}\right.}}^{\text {Cayley }} \backslash\left\{\mathbb{G}^{(m)}\right\}
$$

has property $(\mathrm{T})$. Then every group in the Cayley closure $\overline{\left\{\mathbb{G}^{(m)}\right\}}$ Cayley has property (T). By Lemma 4.2, there exists a decomposition of indices $\mathbb{N}=I_{1} \sqcup I_{2} \sqcup \cdots \sqcup I_{n}$ such that $\mathbb{G}^{(m)}$ is a quotient of $\mathbb{P}_{i}$ for every $m \in I_{i}$. By Corollary 2.11, it suffices to show that each $X^{(i)}=\bigsqcup_{m \in I_{i}} \operatorname{Cay}\left(\mathbb{G}^{(m)}\right)$ has geometric property $(\mathrm{T})$. The group homomorphisms $\phi^{(m)}: P_{i} \rightarrow G^{(m)}, m \in I_{i}$, induce the $*$-homomorphism

$$
\phi_{i}: \mathbb{C}\left[P_{i}\right] \rightarrow \prod_{m \in I_{i}} \mathbb{C}\left[G^{(m)}\right] \subset \mathrm{C}_{\mathrm{u}, \max }^{*}\left[X^{(i)}\right]
$$

which sends the Laplacian $\Delta_{i} \in \mathbb{C}\left[P_{i}\right]$ of the marked group $\mathbb{P}_{i}$ to the Laplacian of $X^{(i)}$. Thus Proposition 2.10 implies that $X^{(i)}$ has geometric property $(\mathrm{T})$.

\section{Remarks}

\section{Uniformity of spectral gaps}

Let $v(G, S)$ be the infimum of strictly positive spectra of the discrete Laplacian $\Delta_{\mathbb{G}}$ in $\mathrm{C}_{\max }^{*}[G]$.

Proposition 5.1 Let $K$ be a nonempty closed subset of $\mathcal{G}(k)$ consisting of groups with Kazhdan's property $(\mathrm{T})$. Then there exists a strictly positive number $v$ such that $v \leq v(G, S)$ for every $(G, S) \in K$.

Proof By Lemma 4.2, there exist finitely many marked groups $\mathbb{P}_{1}, \mathbb{P}_{2}, \ldots, \mathbb{P}_{n}$ with property (T) such that every member $\mathbb{G}$ of $K$ is a quotient of one of $\mathbb{P}_{i}$. Since every representation of $G$ provides a representation of $P_{i}$, we have $0<\min _{i} v\left(\mathbb{P}_{i}\right) \leq v(\mathbb{G})$. 
Proposition 5.1 roughly states that for a (nonempty) compact set $K$ in $\mathcal{G}(k)$, uniformity concerning property (T) is automatic once all of the members of $K$ have property (T). In [16], two of the authors have showed that, concerning amenability, a similar phenomenon occurs; see [16, proof of Theorem 5.1]. On the other hand, two of the authors have revealed that concerning a-T-menability, the uniformity is not automatically guaranteed. For details, see the paper that is part II of this series [17]. In [15], we further study the spectral gap and Kazhdan constant. These constants define functions on the space of marked groups. We will prove that these are lower semi-continuous functions on $\mathcal{G}(k)$. (Note that for spectral gaps, it can be also derived from the main result of [20]. See Theorem 3.1.) We also study spectral gaps and Kazhdan-type constants for isometric actions on certain metric spaces, including uniformly convex Banach spaces and complete $\mathrm{CAT}(0)$ spaces.

\section{Uniformity on amenability}

Proposition 5.2 Let $\left\{\left(G^{(m)}, s^{(m)_{1}}, \ldots, s^{(m)_{k}}\right)\right\}_{m \in \mathbb{N}}$ be a sequence of amenable $k$ marked groups. Suppose that the sequence converges to a $k$-marked group $\mathbb{G}^{(\infty)}$. Then the following conditions are equivalent:

(1) The metric space $\bigsqcup_{m} \operatorname{Cay}\left(\mathbb{G}^{(m)}\right)$ has property A of Yu.

(2) The limit group $\mathbb{G}^{(\infty)}$ is amenable.

(3) There exists an amenable $k$-marked group $\mathbb{P}$ such that for every $m$, the group $\mathbb{G}^{(m)}$ is a quotient of $\mathbb{P}$.

Proof By [16, Theorem 5.1], conditions (1) and (2) are equivalent. Suppose condition (3) holds. The set $\mathcal{Q}_{\mathbb{P}}$ forms a closed subset in the space of $k$-marked groups and consists of amenable groups. It follows that the closure $\frac{\left.\mathbb{G}^{(m)}\right\}_{m}}{\text { Cayley }}$ is contained in $\mathcal{Q}_{\mathbb{P}}$. In particular, condition (2) holds. Conversely, suppose condition (2) holds. Define a $k$-marked group $\left(P, \gamma_{1}, \ldots, \gamma_{k}\right)$ by the subgroup of $\prod_{m=1}^{\infty} G^{(m)}$ generated by $\gamma_{1}=\left(s_{1}^{(m)}\right)_{m}, \ldots, \gamma_{k}=\left(s_{k}^{(m)}\right)_{m}$. Each $\mathbb{G}^{(m)}$ is a quotient of $\mathbb{P}$. Note that the limit group $\mathbb{G}^{(\infty)}$ is also a quotient of $\mathbb{P}$. Let $H$ be the kernel of the quotient map $P \rightarrow G^{(\infty)}$. The group $H$ is equal to

$$
\left\{\left(g^{(m)}\right)_{m} \in P \mid \text { there exists } M \in \mathbb{N} \text { such that for all } m \geq M, g^{(m)}=1_{G^{(m)}}\right\} .
$$

The subgroup $H_{M}$ defined by

$$
\left\{\left(g^{(m)}\right)_{m} \in P \mid \text { for all } m \geq M, g^{(m)}=1_{G^{(m)}}\right\}
$$

is amenable, since $\bigoplus_{m=1}^{M} G^{(m)}$ is amenable. Since $H$ is an increasing union of amenable groups $H_{M}$, it is amenable. Since $P$ is in the middle of the short exact 
sequence

$$
1 \rightarrow H \rightarrow P \rightarrow G^{(\infty)} \rightarrow 1,
$$

$P$ is also amenable. Therefore condition (3) holds.

\section{Cohomological property (T)}

By the well-known Delorme-Guichardet theorem [1, Theorem 2.12.4], property $(\mathrm{T})$ for a (locally compact $\sigma$-compact) group $G$ can be characterized by the vanishing of the first cohomology group $H^{1}(G, \mathcal{H})$ of every unitary $G$-module $\mathcal{H}$. In this section, we study this phenomenon for coarse spaces. Namely, we will introduce cohomological property $(\mathrm{T})$ for uniformly locally finite metric spaces and prove that it implies geometric property $(\mathrm{T})$. For this purpose, we develop a cohomology theory for such spaces, in analogy with the cohomology theory for group actions. See [6; 8, Chapter 8; $19 ; 22 ; 23$, Chapter 5] for relevant results. We first work purely algebraically. So we consider a unital algebra $\mathcal{A}$ (over $\mathbb{C}$ ) together with an "augmentation" map $\omega$ from $\mathcal{A}$ onto a unital subalgebra $\mathcal{D} \subset \mathcal{A}$ that satisfies $\left.\omega\right|_{\mathcal{D}}=\mathrm{id}_{\mathcal{D}}$ and $\omega(a b)=\omega(a \omega(b))$ for $a, b \in \mathcal{A}$. (Although we are content with the unital setting, one may also want to look at a non-unital algebra $\mathcal{A}$ and a unital subalgebra $\mathcal{D}$ in the multiplier of $\mathcal{A}$.) It follows that $\mathcal{L}:=\operatorname{ker} \omega$ is a left ideal of $\mathcal{A}$. Prototypical examples are group algebras $\mathbb{C}[G]$ and the unit character $\omega: \mathbb{C}[G] \rightarrow \mathbb{C} \subset \mathbb{C}[G]$; and the algebraic uniform Roe algebras $\mathbb{C}_{\mathrm{u}}[X]$ of a uniformly locally finite metric space $X$ and $\omega: \mathbb{C}_{\mathrm{u}}[X] \rightarrow \ell_{\infty}(X) \subset \mathbb{C}_{\mathrm{u}}[X]$, given by $\omega(a)(x)=\sum_{y} a_{x, y}$ for $a=\left[a_{x, y}\right]_{x, y \in X} \in \mathbb{C}_{\mathrm{u}}[X]$ (this augmentation map $\omega$ is denoted by $\Phi$ in [31]).

For a given left $\mathcal{A}$-module $\mathcal{M}$, we define the cohomology groups $H^{n}(\mathcal{A}, \mathcal{M})$ to be the relative Ext-groups of the $\mathcal{D}$-algebra $\mathcal{A}$ with coefficients in $\mathcal{M}$ relative to $\mathcal{L}$. Namely, $H^{n}(\mathcal{A}, \mathcal{M})=\operatorname{ker} d_{n+1} / \operatorname{ran} d_{n}$ for the cochain complex

$\mathcal{M} \stackrel{d_{1}}{\longrightarrow} \operatorname{Hom}_{\mathcal{D}}(\mathcal{L}, \mathcal{M}) \stackrel{d_{2}}{\longrightarrow} \operatorname{Hom}_{\mathcal{D}}\left(\mathcal{A} \otimes_{\mathcal{D}} \mathcal{L}, \mathcal{M}\right)$

$$
\stackrel{d_{3}}{\longrightarrow} \cdots \stackrel{d_{n}}{\longrightarrow} \operatorname{Hom}_{\mathcal{D}}(\overbrace{\mathcal{A} \otimes_{\mathcal{D}} \cdots \otimes_{\mathcal{D}} \mathcal{A}}^{n-1} \otimes_{\mathcal{D}} \mathcal{L}, \mathcal{M}) \stackrel{d_{n+1}}{\longrightarrow} \cdots,
$$

where $\operatorname{Hom}_{\mathcal{D}}$ is the space of $\mathcal{D}$-module maps and

$$
\begin{aligned}
& \left(d_{n} \theta\right)\left(a_{1} \otimes \cdots \otimes a_{n}\right) \\
& \quad=a_{1} \theta\left(a_{2} \otimes \cdots \otimes a_{n}\right)+\sum_{i=1}^{n-1}(-1)^{i} \theta\left(a_{1} \otimes \cdots \otimes a_{i-1} \otimes a_{i} a_{i+1} \otimes a_{i+2} \otimes \cdots \otimes a_{n}\right) .
\end{aligned}
$$

For example, 1-cocycles are $\mathcal{A}$-module maps $\theta: \mathcal{L} \rightarrow \mathcal{M}$ and 1-coboundaries are those given by $\mathcal{L} \ni a \mapsto a v \in \mathcal{M}$ for some $v \in \mathcal{M}$. In the case where $\mathcal{A}=\mathbb{C}[G]$ and 
$\omega: \mathbb{C}[G] \rightarrow \mathbb{C} \subset \mathbb{C}[G]$ is the unit character, it is not difficult to check that $H^{n}(\mathcal{A}, \mathcal{M})$ is nothing but the group cohomology $H^{n}(G, \mathcal{M})$. In the case where $\mathcal{A}=\mathbb{C}_{\mathrm{u}}[X]$, we will denote $H^{n}(\mathcal{A}, \mathcal{M})$ by $H^{n}(X, \mathcal{M})$.

Proposition 6.1 Let $\mathcal{D}$ be a unital algebra on which a group $G$ acts and $\mathcal{A}=\mathcal{D} \rtimes G$ be the algebraic crossed product, together with the augmentation map $\omega: \mathcal{A} \rightarrow \mathcal{D}$ given by

$$
\omega\left(\sum_{g \in G} a_{g} g\right)=\sum_{g \in G} a_{g} .
$$

Then for every left $\mathcal{A}$-module $\mathcal{M}$, the homomorphisms $\iota_{*}: H^{n}(\mathcal{A}, \mathcal{M}) \rightarrow H^{n}(G, \mathcal{M})$ induced by the inclusion $\iota: \mathbb{C}[G] \hookrightarrow \mathcal{A}$ are isomorphisms.

Proof We note that $G$ (resp. $\left\{g-1_{G}: g \in G \backslash\left\{1_{G}\right\}\right\}$ ) is a basis for the left $\mathcal{D}$-module $\mathcal{A}($ resp. $\mathcal{L}:=\operatorname{ker} \omega)$. Also note that $\mathcal{L}[G]:=\operatorname{ker} \omega \cap \mathbb{C}[G]=\operatorname{span}_{\mathbb{C}}\left\{g-1_{G}\right\}$. It follows that the linear map

$$
\mathcal{D} \otimes_{\mathbb{C}} \overbrace{\mathbb{C}[G] \otimes_{\mathbb{C}} \cdots \otimes_{\mathbb{C}} \mathbb{C}[G]}^{n-1} \otimes_{\mathbb{C}} \mathcal{L}[G] \rightarrow \overbrace{\mathcal{A} \otimes_{\mathcal{D}} \cdots \otimes_{\mathcal{D}} \mathcal{A}}^{n-1} \otimes_{\mathcal{D}} \mathcal{L}
$$

given by $a \otimes \xi_{1} \otimes \cdots \otimes \xi_{n} \mapsto\left(a \xi_{1}\right) \otimes \xi_{2} \otimes \cdots \otimes \xi_{n}$ is a $\mathcal{D}$-module isomorphism. Hence, every $n$-cocycle $\theta$ for $\mathbb{C}[G]$ uniquely extends to an $n$-cocycle for $\mathcal{A}$. This gives rise to the inverse of $\iota_{*}$ and so $\iota_{*}$ is an isomorphism.

Corollary 6.2 Let $G$ be a group and $X=\operatorname{Cay}(G)$. Then, for every left $\mathbb{C}_{\mathrm{u}}[X]-$ module $\mathcal{M}$, there are canonical isomorphisms $H^{n}(X, \mathcal{M}) \cong H^{n}(G, \mathcal{M})$.

Proof Apply Proposition 6.1 to $\mathbb{C}_{\mathrm{u}}[X] \cong \ell_{\infty}(X) \rtimes G$.

Here we recover a result of Pansu [22] that vanishing of $L^{2}$-Betti numbers is a coarse invariant.

Corollary 6.3 Let $G_{1}$ and $G_{2}$ be groups that are coarsely equivalent. Then, for every $n$ one has $\beta_{n}\left(G_{1}\right)=0 \Leftrightarrow \beta_{n}\left(G_{2}\right)=0$. Here $\beta_{n}(G)$ denotes the $n^{\text {th }} L^{2}-B e t t i$ number of $G$.

Proof If one of $G_{1}$ and $G_{2}$ is amenable, then both are amenable [23, Proposition 3.3.5] and have zero $L^{2}$-Betti numbers by the Cheeger-Gromov theorem [14, Theorem 6.3.7]. So we assume that $G_{1}$ and $G_{2}$ are not amenable. By [28], there is a bijective coarse equivalence between $G_{1}$ and $G_{2}$. (This may not be true when the groups are amenable [5]; still their algebraic uniform Roe algebras are Morita equivalent.) Thus, for $X:=$ $\operatorname{Cay}\left(G_{1}\right)=\operatorname{Cay}\left(G_{2}\right)$, Corollary 6.2 yields a canonical isomorphism $H^{n}\left(G_{1}, \ell_{2}(X)\right) \cong$ 
$H^{n}\left(G_{2}, \ell_{2}(X)\right)$ that preserves the topologies also. Here for a topological $G$-module $\mathcal{M}$, the topology on $H^{n}(G, \mathcal{M})$ is induced from the pointwise convergence topology on the space of cochains. Since $\beta_{n}(G)=0$ if and only if $H^{n}\left(G, \ell_{2}(G)\right)=\overline{\{0\}}$, we are done.

Definition 6.4 Let $X$ be a uniformly locally finite metric space. We say $X$ has cohomological property $(\mathrm{T})$ if $H^{1}(X, \mathcal{H})=0$ for every $*$-representation of the algebraic uniform Roe algebra $\mathbb{C}_{\mathrm{u}}[X]$ on a Hilbert space $\mathcal{H}$.

We denote the augmentation left ideal $\operatorname{ker}\left(\omega: \mathbb{C}_{\mathrm{u}}[X] \rightarrow \ell_{\infty}(X)\right)$ by $\mathcal{L}_{\mathrm{u}}[X]$. Suppose that a 1 -cocycle $\theta: \mathcal{L}_{\mathrm{u}}[X] \rightarrow \mathcal{H}$ is given. Then $b(t):=\theta(t-\omega(t))$ is a 1-cocycle for the pseudo-group of partial translations $t$ in $\mathbb{C}_{\mathrm{u}}[X]$. Namely, it satisfies the cocycle identity $b(s t)=b\left(\left.s\right|_{\operatorname{ran}(t)}\right)+\pi\left(\left.s\right|_{\operatorname{ran}(t)}\right) b(t)$ for $s$ and $t$. The following criterion of 1 -coboundaries is handy, as in the case of group 1-cocycles.

Lemma 6.5 A 1-cocycle $\theta: \mathcal{L}_{\mathrm{u}}[X] \rightarrow \mathcal{H}$ is a 1-coboundary if and only if

$$
\sup _{t}\|\theta(t-\omega(t))\|<\infty
$$

where the supremum is taken all over the partial (resp. full) translations $t$.

Proof We only have to prove the "if" part. Let $\Gamma_{X}$ be the group of full translations in $\mathbb{C}_{\mathrm{u}}[X]$. Since the cocycle $\theta$ is bounded, there is $v \in \mathcal{H}$ such that $\theta(t-1)=\pi(t) v-v$ for all $t \in \Gamma_{X}$, by [1, Proposition 2.2.9]. But since $\mathcal{L}_{\mathrm{u}}[X]=\operatorname{span}\left(\ell_{\infty}(X)\left\{t-1: t \in \Gamma_{X}\right\}\right)$ (see Lemma 2.5), one has $\theta(a)=\pi(a) v$ for all $a \in \mathcal{L}_{\mathrm{u}}[X]$.

It is proved in [31] that geometric property $(\mathrm{T})$ is a coarse invariant. The same is true for the cohomological property $(\mathrm{T})$.

Theorem 6.6 Let $X$ and $Y$ be uniformly locally finite metric spaces that are coarsely equivalent. Then $X$ has cohomological property (T) if and only if $Y$ has it.

Proof We first prove that if an inclusion $X \hookrightarrow Y$ is a coarse equivalence and $X$ has cohomological property (T), then so does $Y$. Since $Y$ is uniformly locally finite, there is a finite family $\left\{f_{i}\right\}$ of partial translations such that $\operatorname{dom}\left(f_{i}\right) \subset X$ and $\bigcup_{i} \operatorname{ran}\left(f_{i}\right)=Y$. It is not too difficult to see that every partial translation on $Y$ is a concatenation of partial translations of the form $f_{j} \circ s_{i j} \circ f_{i}^{-1}$, where the $s_{i j}$ are partial translations on $X$. Now suppose that a 1 -cocycle $\theta: \mathcal{L}_{\mathrm{u}}[Y] \rightarrow \mathcal{H}$ is given. By cohomological property (T) of $X$, the pseudo-group cocycle $b(s):=\theta(s-\omega(s))$ is uniformly bounded on partial translations $s$ of $X$. By the cocycle identity, $b\left(f_{j} \circ s \circ f_{i}^{-1}\right)$ is also uniformly bounded 
for every $i$ and $j$. It follows that $b$ is bounded on partial translations of $Y$, and by Lemma 6.5 it is a 1 -coboundary. This proves that $Y$ has cohomological property (T).

In view of Lemma 2.1, it remains show that cohomological property (T) of $X \times \boldsymbol{n}$ implies that of $X$. We identify $\mathbb{C}_{\mathrm{u}}[\boldsymbol{n}]$ with $\mathbb{M}_{n}(\mathbb{C})$ and $\mathbb{C}_{\mathrm{u}}[X \times \boldsymbol{n}]$ with $\mathbb{M}_{n}(\mathbb{C}) \otimes \mathbb{C}_{\mathrm{u}}[X]$ via

$$
\left[a_{(x, i),(y, j)}\right]_{(x, i),(y, j)} \leftrightarrow \sum_{i, j} e_{i j} \otimes\left[a_{(x, i),(y, j)}\right]_{x, y} .
$$

Then, the augmentation map $\omega_{X \times \boldsymbol{n}}$ for $X \times \boldsymbol{n}$ is of the form $\omega_{X \times \boldsymbol{n}}=\omega_{\boldsymbol{n}} \otimes \omega$ where $\omega_{\boldsymbol{n}}\left(\left[a_{i, j}\right]_{i, j}\right)=\left[\delta_{i, j} \sum_{k} a_{i, k}\right]_{i, j}$ on $\mathbb{M}_{n}(\mathbb{C})$. It follows that $\sum_{i, j} e_{i j} \otimes a_{i, j} \in \mathcal{L}_{\mathrm{u}}[X \times \boldsymbol{n}]$ implies $\sum_{j} a_{i, j} \in \mathcal{L}_{\mathrm{u}}[X]$ for every $i$. Let a $*$-representation $\pi: \mathbb{C}_{\mathrm{u}}[X] \rightarrow \mathbb{B}(\mathcal{H})$ and a 1 -cocycle $\theta: \mathcal{L}_{\mathrm{u}}[X] \rightarrow \mathcal{H}$ be given. Then we define a 1-cocycle $\theta_{n}: \mathcal{L}_{\mathrm{u}}[X \times \boldsymbol{n}] \rightarrow \mathcal{H}^{\oplus n}$ for the $*$-representation $\pi_{n}=\mathrm{id} \otimes \pi$ of $\mathbb{M}_{n}(\mathbb{C}) \otimes \mathbb{C}_{\mathrm{u}}[X]$ on $\mathcal{H}^{\oplus n}$ by

$$
\theta_{n}\left(\sum_{i, j} e_{i j} \otimes a_{i, j}\right)=\left(\theta\left(\sum_{j} a_{i, j}\right)\right)_{i=1}^{n} .
$$

It is indeed a 1 -cocycle and hence there is $\widetilde{v}=\left(v_{i}\right)_{i=1}^{n} \in \mathcal{H}^{\oplus n}$ such that $\theta_{n}(\widetilde{a})=\pi_{n}(\widetilde{a}) \widetilde{v}$ for all $\tilde{a} \in \mathcal{L}_{\mathrm{u}}[X \times \boldsymbol{n}]$. It follows that $\theta(a)=\pi(a) v_{1}$ for all $a \in \mathcal{L}_{\mathrm{u}}[X]$, and $\theta$ is a 1 -coboundary.

The following finishes the proof of Theorem 1.1.

Theorem 6.7 For a uniformly locally finite metric space $X$, one has the following.

- Cohomological property (T) implies geometric property (T).

- If the coarse structure of $X$ is induced by a property (T) group $G$ (ie, $G=\langle S\rangle$ acts on $X$ in such a way that $\{(x, g x): x \in X, g \in S\}$ is a generating controlled subset), then $X$ has cohomological property (T).

- In case $X$ is a disjoint union of the Cayley graphs of finite $k$-marked groups, $X$ has cohomological property $(\mathrm{T})$ if and only if it has geometric property $(\mathrm{T})$.

Proof Suppose that $X$ does not have geometric property (T). By [31, Proposition 3.8], there are $*$-representations $\pi_{n}$ of $\mathbb{C}_{\mathrm{u}}[X]$ on $\mathcal{H}_{n}$ and unit vectors $\xi_{n} \in\left(\mathcal{H}_{n}\right)_{c}^{\perp}$ such that $\left\|\pi_{n}(a-\omega(a)) \xi_{n}\right\| \leq 4^{-n} \sup _{x, y \in X}\left|a_{x, y}\right|$ for every $a=\left[a_{x, y}\right]_{x, y}$ of propagation at most $n$. Here $\left(\mathcal{H}_{n}\right)_{c}$ is the space of constant vectors, which consists of the vectors in $\mathcal{H}_{n}$ that are annihilated by $\pi_{n}\left(\mathcal{L}_{\mathrm{u}}[X]\right)$. (Remember that our $\omega$ is denoted by $\Phi$ in [31].) Now, we may define a 1 -cocycle $\theta$ from $\mathcal{L}_{\mathrm{u}}[X]$ into $\bigoplus_{n} \mathcal{H}_{n}$ by $\theta(a)=$ $\sum_{n}^{\oplus} 2^{n} \pi_{n}(a) \xi_{n}$. We claim that $\theta$ is not a $1-$ coboundary. For if it were, there would be $\zeta=\sum_{n}^{\oplus} \zeta_{n} \in \bigoplus_{n} \mathcal{H}_{n}$ such that $\theta(a)=\sum^{\oplus} \pi_{n}(a) \zeta_{n}$. This means that $\pi_{n}(a) \zeta_{n}=$ 
$2^{n} \pi_{n}(a) \xi_{n}$ for every $a \in \mathcal{L}_{\mathrm{u}}[X]$, or equivalently, $\zeta_{n}-2^{n} \xi_{n} \in\left(\mathcal{H}_{n}\right)_{c}$. But this implies

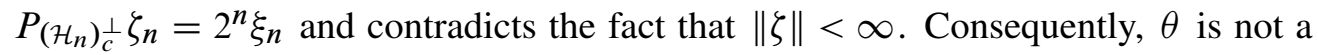
1-coboundary and $X$ does not have cohomological property (T). This proves the first assertion.

The second assertion follows from the observation that every 1-cocycle $\theta: \mathcal{L}_{\mathrm{u}}[X] \rightarrow \mathcal{H}$ is a 1-coboundary for the induced unitary representation of a property (T) group $G$, which implies that $\theta$ is also a $1-$ coboundary for $\mathbb{C}_{\mathrm{u}}[X]$. The last assertion follows from Theorem 1.1:(1) $\Leftrightarrow(2)$, Lemma 4.2, and the previous two assertions.

In fact, by adapting the method of [20], one can prove that cohomological property (T) implies $\Delta^{2}-v \Delta \in \Sigma^{2} \mathbb{C}_{\mathrm{u}}[X]$ for some $v>0$ (see Theorem 3.1). The following is a characterization of Cayley metric spaces $\operatorname{Cay}(G)$ having cohomological property $(\mathrm{T})$. Recall that a unitary $G$-representation $\pi$ is said to be weakly regular if it is weakly contained in the regular representation on $\ell_{2}(G)$.

Proposition 6.8 Let $X=\operatorname{Cay}(G)$ be the Cayley metric space and assume that $X$ has property A (or equivalently, $G$ is exact). Then, $X$ has cohomological property (T) if and only if $H^{1}(G, \mathcal{H})=0$ for every weakly regular unitary $G$-representation $\mathcal{H}$.

Proof Since $X=\operatorname{Cay}(G)$ has property A, every *-representation of $\mathbb{C}_{\mathrm{u}}[X]$ is weakly contained in the regular representation on $\ell_{2}(X)$ by [26, Proposition 1.3]. Thus the "if" part of the proposition follows from Corollary 6.2. Conversely, suppose that $H^{1}(G, \mathcal{H}) \neq 0$ for a weakly regular $G$-representation $\pi$ on $\mathcal{H}$. We view $\pi$ as a $*$-homomorphism from the reduced group $\mathrm{C}^{*}$-algebra $\mathrm{C}_{\lambda}^{*}(G)$ into $\mathbb{B}(\mathcal{H})$. By Arveson's and Stinespring's theorems applied in tandem to $\mathrm{C}_{\lambda}^{*}(G) \subset \mathrm{C}_{\mathrm{u}}^{*}[X]$, there are a Hilbert space $\hat{\mathcal{H}} \supset \mathcal{H}$ and a $*$-representation $\widehat{\pi}: \mathrm{C}_{\mathrm{u}}^{*}[X] \rightarrow \mathbb{B}(\hat{\mathcal{H}})$ such that $\left.\hat{\pi}(g)\right|_{\mathcal{H}}$ coincides with the original $\pi(g)$ for every $g \in G$. Since $H^{1}(G, \widehat{\mathcal{H}}) \supset H^{1}(G, \mathcal{H}) \neq 0$, one has $H^{1}(X, \widehat{\mathcal{H}}) \neq 0$ by Corollary 6.2 .

It follows that $\operatorname{Cay}\left(F_{2}\right)$ has geometric property $(\mathrm{T})$ [31, Corollary 6.5], but not cohomological property $(\mathrm{T})$ (since $H^{1}\left(F_{2}, \ell_{2}\left(F_{2}\right)\right) \neq 0$ ). Lattices in $\operatorname{SL}(2, \mathbb{C})$ also do not have cohomological property (T) [9, Exemple 3] although they have zero first $L^{2}$-Betti numbers [14, Theorem 4.1]. These examples show that cohomological property (T) is in general strictly stronger than geometric property $(\mathrm{T})$. The authors do not know whether these properties are equivalent for a disjoint union of finite metric spaces of bounded geometry. There are many Cayley metric spaces that have cohomological property $(\mathrm{T})$, besides those come from property (T) groups, eg $\operatorname{Cay}\left(F_{2} \times F_{2}\right)$. Indeed, it is well known that $H^{1}\left(G_{1} \times G_{2}, \mathcal{H}\right)=0$ for every non-amenable group $G_{i}$ and every weakly regular representation $(\pi, \mathcal{H})$. We sketch the proof of this fact 
for the reader's convenience. Let $b: G_{1} \times G_{2} \rightarrow \mathcal{H}$ be a 1-cocycle, ie it satisfies $b(g h)=b(g)+\pi(g) b(h)$ for every $g, h \in G_{1} \times G_{2}$. Thus, for every $g_{1} \in G_{1}$ and $g_{2} \in G_{2}$, one has $\left(\pi\left(g_{2}\right)-1\right) b\left(g_{1}\right)=\left(\pi\left(g_{1}\right)-1\right) b\left(g_{2}\right)$. Since $G_{i}$ is not amenable, $\pi$ does not weakly contain the trivial representation, or equivalently, there is a finite subset $S_{i} \subset G_{i}$ and $C>0$ such that $\|v\| \leq C\left\|\sum_{g \in S_{i}}(\pi(g)-1) v\right\|$ holds for all $v \in \mathcal{H}$. This implies that $b$ is bounded on each of the $G_{i}$ and hence on $G_{1} \times G_{2}$. Such a 1-cocycle is a 1 -coboundary [1, Proposition 2.2.9].

\section{On the structure of maximal uniform Roe algebras}

In this section, we develop the representation theory of the maximal uniform Roe algebras of a (coarse) disjoint union. For the reader's convenience, we recall the basic properties of $C(K)$ - $\mathrm{C}^{*}$-algebras [11] in the unital setting. Let $K$ be a compact Hausdorff space. A unital $\mathrm{C}^{*}$-algebra $A$ is called a $C(K)-\mathrm{C}^{*}$-algebra if it comes together with a $*$-homomorphism $\theta$ from $C(K)$ into the center of $A$. We will omit writing $\theta$ as if $C(K)$ is a subalgebra of $A$. For each $t \in K$, let $I_{t}=\overline{C_{0}(K \backslash\{t\}) A}$ be the corresponding ideal of $A$ (in fact $I_{t}=C_{0}(K \backslash\{t\}) A$ by Cohen's factorization theorem), and denote by $\pi_{t}: A \rightarrow A / I_{t}=: A_{t}$ the corresponding quotient. Then, every irreducible representation of $A$ factors through some $\pi_{t}$, since its restriction to $C(K)$ is a character associated with some point $t \in K$. It follows that the representation $A \ni a \mapsto \prod_{t} \pi_{t}(a) \in \prod_{t} A_{t}$ is faithful. In particular, $\operatorname{Sp}(a)=\overline{\bigcup_{t} \operatorname{Sp}\left(\pi_{t}(a)\right)}$ for every $a \in A$. Each $A_{t}$ is called a fiber of $A$. A $*$-homomorphism $\sigma: A \rightarrow B$ between $C(K)-C^{*}$-algebras $A$ and $B$ is simply called a morphism if its restriction to $C(K)$ is the identity map. Such a morphism naturally induces $*$-homomorphisms $\sigma_{t}: A_{t} \rightarrow B_{t}$ on the fibers. Note that a morphism $\sigma: A \rightarrow B$ is injective if and only if it is the case for each fiber $\sigma_{t}: A_{t} \rightarrow B_{t}$. In particular, a $C(K)-C^{*}$-algebra $A$ is nuclear if and only if all fibers $\left\{A_{t}\right\}_{t}$ are nuclear.

Now, we consider a $*$-algebra $\mathcal{B}$ containing $C(K)$ in its center. Then its universal enveloping $\mathrm{C}^{*}$-algebra $A=\mathrm{C}^{*}(\mathcal{B})$ is a $C(K)-\mathrm{C}^{*}$-algebra. For each $t \in K$, the ideal $\mathcal{J}_{t}=C_{0}(K \backslash\{t\}) \mathcal{B}$ is dense in the ideal $I_{t}$ of $A$. It is not hard to see that $A_{t}=A / I_{t}$ is the universal enveloping $C^{*}$-algebra of $\mathcal{B}_{t}:=\mathcal{B} / \mathcal{J}_{t}$.

Let $\left\{\left(G^{(m)}, s_{1}^{(m)}, \ldots, s_{k}^{(m)}\right)\right\}_{m}$ be a sequence of $k$-marked groups and denote by $\sigma_{m}$ the corresponding homomorphism from $F_{k}=\left\langle s_{1}, \ldots, s_{k}\right\rangle$ onto $G^{(m)}$ that maps $s_{i}$ to $s_{i}^{(m)}$. Let $X=\bigsqcup_{m} \operatorname{Cay}\left(G^{(m)}, s_{1}^{(m)}, \ldots, s_{k}^{(m)}\right)$ be the disjoint union. For $g \in F_{k}$, let $v_{g}$ denote the element in $\mathbb{C}_{\mathrm{u}}[X]$ that is represented by the kernel

$$
\left(v_{g}\right)(x, y)= \begin{cases}1 & \text { if } x, y \in G^{(m)} \text { and } x=\sigma_{m}(g) y, \\ 0 & \text { otherwise }\end{cases}
$$


and note that

$$
\mathbb{C}_{\mathrm{u}}[X]=\left\{\sum_{g \in F_{k}} \xi_{g} v_{g} \mid \xi_{g} \in \ell_{\infty}(X) \text { are zero for all but finitely many } g\right\} .
$$

The center of the algebraic uniform Roe algebra $\mathbb{C}_{\mathrm{u}}[X]$ consists of those functions in $\ell_{\infty}(X)$ that are constant on each of the $G^{(m)}$, and so it is canonically isomorphic to $\ell_{\infty}(\mathbb{N})$. We recall that the Gelfand spectrum of $\ell_{\infty}(\mathbb{N})$ is the Stone-Čech compactification $\beta \mathbb{N}$ of $\mathbb{N}$, and $\ell_{\infty}(\mathbb{N})$ is $*$-isomorphic to $C(\beta \mathbb{N})$. Thus, the maximal uniform Roe algebra $\mathrm{C}_{\mathrm{u}, \max }^{*}[X]$ is a $C(\beta \mathbb{N})-\mathrm{C}^{*}$-algebra. Let us fix an element $\omega \in \beta \mathbb{N}$ for a while, and identify it with the corresponding character $\omega: \ell_{\infty}(\mathbb{N}) \rightarrow \mathbb{C}$. We will denote $\omega(\xi)$ also by $\lim _{\omega} \xi(m)$. We still abuse the notation and denote the corresponding ultrafilter by $\omega$, too. Namely, we identify $\omega$ with the family of those subsets $E \subset \mathbb{N}$ such that $\omega\left(1_{E}\right)=1$. Here we recall that an ultrafilter is a family of non-empty subsets that satisfies the finite intersection property and the maximality condition that $E \notin \omega$ implies $(\mathbb{N} \backslash E) \in \omega$. For example, $n \in \mathbb{N}$ is identified with the principal character $\ell_{\infty}(\mathbb{N}) \ni \xi \mapsto \xi(n)$ and with the principal ultrafilter consisting of the subsets $E \subset \mathbb{N}$ that contain $n$. By the universality of the Stone-Čech compactification, the map $\mathbb{N} \ni m \mapsto \mathbb{G}^{(m)} \in \mathcal{G}(k)$ extends to a continuous map $\beta \mathbb{N} \ni \omega \mapsto \mathbb{G}^{(\omega)} \in \mathcal{G}(k)$. We note that

$$
\mathbb{G}^{(\omega)}=\mathbb{F}_{k} /\left\{w \in F_{k}:\left\{m \in \mathbb{N} \mid \sigma_{m}(w)=1\right\} \in \omega\right\}
$$

as a $k$-marked group, with the corresponding homomorphism denoted by $\sigma_{\omega}: F_{k} \rightarrow$ $G^{(\omega)}$. To see the relation between $G^{(\omega)}$ and $\mathbb{C}_{\mathrm{u}}[X]_{\omega}$, let us observe that

$$
\begin{gathered}
C_{0}(\beta \mathbb{N} \backslash\{\omega\}) \ell_{\infty}(X)=\left\{\left(\xi^{(m)}\right)_{m=1}^{\infty} \in \prod_{m=1}^{\infty} \ell_{\infty}\left(G^{(m)}\right) \mid \lim _{\omega}\left\|\xi^{(m)}\right\|=0\right\}, \\
C_{0}(\beta \mathbb{N} \backslash\{\omega\}) \mathbb{C}_{\mathrm{u}}[X]=\left\{a=\left[a_{x, y}\right]_{x, y} \in \mathbb{C}_{\mathrm{u}}[X]\left|\lim _{\omega} \sup _{x, y \in G^{(m)}}\right| a_{x, y} \mid=0\right\} .
\end{gathered}
$$

Hence, $\pi_{\omega}\left(v_{g}\right)=1$ in $\mathbb{C}_{\mathrm{u}}[X]_{\omega} \Leftrightarrow 1-v_{g} \in C_{0}(\beta \mathbb{N} \backslash\{\omega\}) \mathbb{C}_{\mathrm{u}}[X] \Leftrightarrow \sigma_{\omega}(g)=1$. This means that $g \mapsto \pi_{\omega}\left(v_{g}\right)$ gives rise to an inclusion $G^{(\omega)} \hookrightarrow \mathbb{C}_{\mathrm{u}}[X]_{\omega}$. Moreover, $\mathbb{C}_{\mathrm{u}}[X]_{\omega}$ is canonically isomorphic to the algebraic crossed product $\ell_{\infty}(X)_{\omega} \rtimes G^{(\omega)}$. Here $\ell_{\infty}(X)_{\omega}:=\ell_{\infty}(X) /\left(C_{0}(\beta \mathbb{N} \backslash\{\omega\}) \ell_{\infty}(X)\right)$, which is nothing but the ultraproduct $\mathrm{C}^{*}-$ algebra of the $\ell_{\infty}\left(G^{(m)}\right)$. We denote by $\xi^{(\omega)}$ the element in $\ell_{\infty}(X)_{\omega}$ that corresponds to $\xi=\left(\xi^{(m)}\right)_{m=1}^{\infty} \in \prod_{m=1}^{\infty} \ell_{\infty}\left(G^{(m)}\right)$. For $g \in G^{(\omega)}$, let $u_{g}$ denote the corresponding element in the maximal (or full) crossed product $\mathrm{C}^{*}$-algebra $\ell_{\infty}(X)_{\omega} \rtimes_{\max } G^{(\omega)}$.

Gathering all the above discussions, we have followed due process to ensure the canonicality of the canonical maps. 
Theorem 7.1 Let $X=\bigsqcup_{m \in \mathbb{N}} \operatorname{Cay}\left(G^{(m)}, s_{1}^{(m)}, \ldots, s_{k}^{(m)}\right)$ be as above. With the above notation, the maximal uniform Roe algebra $\mathrm{C}_{\mathrm{u}, \max }^{*}[X]$ is a $C(\beta \mathbb{N})-\mathrm{C}^{*}$-algebra and for each $\omega \in \beta \mathbb{N}$ there is a canonical $*$-isomorphism

$$
\mathrm{C}_{\mathrm{u}, \max }^{*}[X]_{\omega} \cong \ell_{\infty}(X)_{\omega} \rtimes_{\max } G^{(\omega)}
$$

that sends $\sum_{g \in F_{k}} \xi_{g} v_{g}$ in $\mathbb{C}_{\mathrm{u}}[X]$ to $\sum_{g \in F_{k}} \xi_{g}^{(\omega)} u_{\sigma_{\omega}(g)}$.

Corollary 7.2 Use the same notation as in Theorem 7.1 and assume that all the $G^{(m)}$ are amenable. Then for every $\omega \in \beta \mathbb{N}$ there is a canonical $*-$ isomorphism

$$
\pi_{\omega}\left(\mathrm{C}^{*}\left(\left\{v_{g} \mid g \in F_{k}\right\}\right)\right) \cong \mathrm{C}_{\max }^{*}\left[G^{(\omega)}\right] .
$$

Moreover, it is the range of a conditional expectation on $\mathrm{C}_{\mathrm{u}, \max }^{*}[X]_{\omega}$.

Proof Since the $G^{(m)}$ are amenable, there are $G^{(m)}$-invariant states $\mu_{m}$ on $\ell_{\infty}\left(G^{(m)}\right)$, and hence for every $\omega \in \beta \mathbb{N}$ the state $\ell_{\infty}(X) \ni \xi \mapsto \lim _{\omega} \mu_{m}\left(\xi^{(m)}\right)$ gives rise to a $G^{(\omega)}$-invariant state on $\ell_{\infty}(X)_{\omega}$. It follows that the canonical $*$-homomorphism from $\mathrm{C}_{\max }^{*}\left[G^{(\omega)}\right]$ into $\ell_{\infty}(X)_{\omega} \rtimes_{\max } G^{(\omega)}$ is faithful.

This corollary gives an alternative proof that if $X=\bigsqcup_{m} \operatorname{Cay}\left(G^{(m)}, s_{1}^{(m)}, \ldots, s_{k}^{(m)}\right)$ has geometric property (T), then all the groups in the Cayley boundary of $\left\{\mathbb{G}^{(m)}\right\}_{m}$ have property $(\mathrm{T})$. It also recovers a result of [16]: $X$ has property $\mathrm{A}$ if and only if all the groups in the Cayley boundary are amenable.

On this occasion, we clarify the relation between the two different notions of a disjoint union and their associated maximal uniform Roe algebras. So, let $\bigsqcup_{m} X^{(m)}$ (resp. $\bigsqcup_{m} X^{(m)}$ ) be the disjoint union (resp. coarse disjoint union) of a given sequence $\left\{X^{(m)}\right\}_{m}$ of metric spaces. Thus, as a set, $X:=\bigsqcup_{m} X^{(m)}=\bigsqcup_{m} X^{(m)}$, and as a coarse space, $\mathbb{C}_{\mathrm{u}}\left[\bigsqcup_{m} X^{(m)}\right]=\mathbb{C}_{\mathrm{u}}\left[\bigsqcup_{m} X^{(m)}\right]+\mathbb{K}_{0}$, where $\mathbb{K}_{0}$ is the algebra of the finitely supported kernels on $X$. Since $\mathbb{K}_{0}$ is an ideal of $\mathbb{C}_{\mathrm{u}}\left[\bigsqcup_{m} X^{(m)}\right]$ and it has the unique $\mathrm{C}^{*}$-completion $\mathbb{K}\left(\ell_{2}(X)\right)$, it gives rise to an embedding $\mathbb{K}\left(\ell_{2}(X)\right) \hookrightarrow$ $\mathrm{C}_{\mathrm{u}, \max }^{*}\left[\bigsqcup_{m} X^{(m)}\right]$ as a closed two-sided ideal. There is a conditional expectation $E$ from $\mathbb{C}_{\mathrm{u}}\left[\bigsqcup_{m} X^{(m)}\right]$ onto $\mathbb{C}_{\mathrm{u}}\left[\bigsqcup_{m} X^{(m)}\right]$, given by $E(a)_{x, y}=a_{x, y}$ if $x$ and $y$ belong to the same $X^{(m)}$; else $E(a)_{x, y}=0$.

Proposition 7.3 Let $\left\{X^{(m)}\right\}_{m}$ be a sequence of metric spaces. Then, the canonical embedding of $\mathbb{C}_{\mathrm{u}}\left[\bigsqcup_{m} X^{(m)}\right]$ into $\mathbb{C}_{\mathrm{u}}\left[\bigsqcup_{m} X^{(m)}\right]$ extends to a faithful embedding of $\mathrm{C}_{\mathrm{u}, \max }^{*}\left[\bigsqcup_{m} X^{(m)}\right]$ into $\mathrm{C}_{\mathrm{u}, \max }^{*}\left[\bigsqcup_{m} X^{(m)}\right]$. Hence, one has a canonical identification

$$
\mathrm{C}_{\mathrm{u}, \max }^{*}\left[\bigsqcup_{m} X^{(m)}\right]=\mathrm{C}_{\mathrm{u}, \max }^{*}\left[\bigsqcup_{m} X^{(m)}\right]+\mathbb{K}\left(\ell_{2}(X)\right) .
$$


The conditional expectation $E$ also extends to a faithful, unital and completely positive conditional expectation from $\mathrm{C}_{\mathrm{u}, \max }^{*}\left[\bigsqcup_{m} X^{(m)}\right]$ onto $\mathrm{C}_{\mathrm{u}, \max }^{*}\left[\bigsqcup_{m} X^{(m)}\right]$.

Proof We first note that $J_{0}:=\mathbb{K}_{0} \cap \mathbb{C}_{\mathrm{u}}\left[\bigsqcup_{m} X^{(m)}\right]$ has unique $\mathrm{C}^{*}$-completion $J=$ $\bigoplus_{m} \mathbb{M}_{X^{(m)}}$ (the $C^{*}$-direct sum). Since $\mathbb{C}_{\mathrm{u}}\left[\bigsqcup_{m} X^{(m)}\right] / \mathbb{K}_{0}=\mathbb{C}_{\mathrm{u}}\left[\bigsqcup_{m} X^{(m)}\right] / J_{0}$, one has the commuting diagram

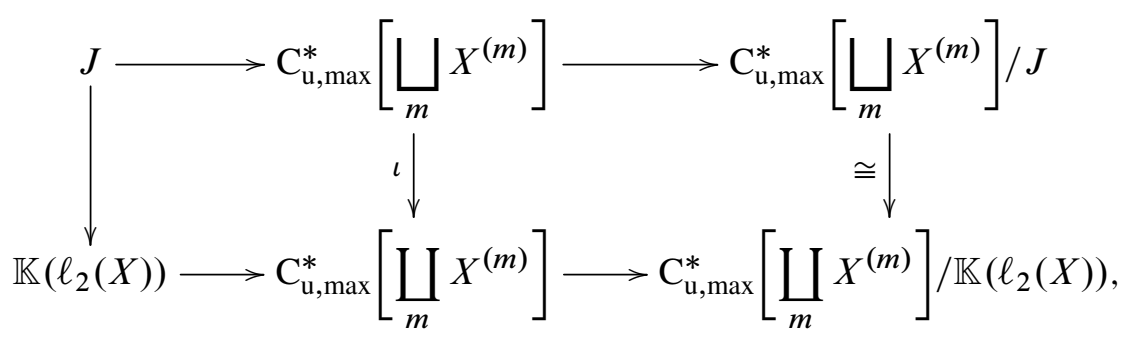

where the right column morphism is an isomorphism. Since the inclusion $\iota$ is injective on $J$, it is injective on $\mathrm{C}_{\mathrm{u}, \max }^{*}\left[\bigsqcup_{m} X^{(m)}\right]$ also. That $E$ extends to a unital and completely positive map on $\mathrm{C}_{\mathrm{u}, \text { max }}^{*}\left[\bigsqcup_{m} X^{(m)}\right]$ follows from the fact that for every $a \in \mathbb{C}_{\mathrm{u}}\left[\bigsqcup_{m} X^{(m)}\right]$ one has

$$
E(a)=\sum_{m=1}^{N} 1_{X^{(m)}} a 1_{X^{(m)}}+\left(1-\sum_{m=1}^{N} 1_{X^{(m)}}\right) a\left(1-\sum_{m=1}^{N} 1_{X^{(m)}}\right)
$$

for a large enough $N$, and in particular $\|E(a)\|_{\max } \leq\|a\|_{\max }$. Since $E$ is faithful on $\mathbb{K}\left(\ell_{2}(X)\right)$ and $\mathrm{C}_{\mathrm{u}, \max }^{*}\left[\bigsqcup_{m} X^{(m)}\right] / \mathbb{K}\left(\ell_{2}(X)\right)$, it is faithful on $\mathrm{C}_{\mathrm{u}, \max }^{*}\left[\bigsqcup_{m} X^{(m)}\right]$.

\section{References}

[1] B Bekka, P de la Harpe, A Valette, Kazhdan's property ( $T)$, New Mathematical Monographs 11, Cambridge Univ. Press (2008) MR2415834

[2] N P Brown, N Ozawa, $C^{*}$-algebras and finite-dimensional approximations, Graduate Studies in Mathematics 88, Amer. Math. Soc. (2008) MR2391387

[3] X Chen, Q Wang, X Wang, Characterization of the Haagerup property by fibred coarse embedding into Hilbert space, Bull. Lond. Math. Soc. 45 (2013) 1091-1099 MR3105001

[4] X Chen, Q Wang, G Yu, The maximal coarse Baum-Connes conjecture for spaces which admit a fibred coarse embedding into Hilbert space, Adv. Math. 249 (2013) 88-130 MR3116568

[5] T Dymarz, Bilipschitz equivalence is not equivalent to quasi-isometric equivalence for finitely generated groups, Duke Math. J. 154 (2010) 509-526 MR2730576 
[6] G Elek, Coarse cohomology and $l_{p}$-cohomology, K-Theory 13 (1998) 1-22 MR1610246

[7] R I Grigorchuk, On the Milnor problem of group growth, Dokl. Akad. Nauk SSSR 271 (1983) 30-33 MR712546 In Russian; translated in Soviet Math. Dokl. 28 (1983) $23-26$

[8] M Gromov, Asymptotic invariants of infinite groups, from: "Geometric group theory, Vol. 2”, (G A Niblo, M A Roller, editors), London Math. Soc. Lecture Note Ser. 182, Cambridge Univ. Press (1993) 1-295 MR1253544

[9] A Guichardet, Sur la cohomologie des groupes topologiques, II, Bull. Sci. Math. 96 (1972) 305-332 MR0340464

[10] P de la Harpe, Topics in geometric group theory, University of Chicago Press (2000) MR1786869

[11] G G Kasparov, Equivariant KK-theory and the Novikov conjecture, Invent. Math. 91 (1988) 147-201 MR918241

[12] A Lubotzky, Discrete groups, expanding graphs and invariant measures, Progress in Mathematics 125, Birkhäuser, Basel (1994) MR1308046

[13] A Lubotzky, A Zuk, On property $(\tau)$, in preparation

[14] W Lück, $L^{2}$-invariants: theory and applications to geometry and $K$-theory, Ergeb. Math. Grenzgeb. 44, Springer, Berlin (2002) MR1926649

[15] M Mimura, Metric Kazhdan constants, in preparation

[16] M Mimura, H Sako, Group approximation in Cayley topology and coarse geometry, I: Coarse embeddings of amenable groups arXiv:1310.4736

[17] M Mimura, H Sako, Group approximation in Cayley topology and coarse geometry, II: Fibered coarse embeddings, in preparation

[18] P W Nowak, G Yu, Large scale geometry, Eur. Math. Soc., Zürich (2012) MR2986138

[19] S Oguni, $L^{2}$-invariants of groups under coarse equivalence and of groupoids under Morita equivalence, in preparation

[20] N Ozawa, Noncommutative real algebraic geometry of Kazhdan's property (T), to appear in J. Inst. Math. Jussieu

[21] N Ozawa, About the Connes embedding conjecture: Algebraic approaches, Jpn. J. Math. 8 (2013) 147-183 MR3067294

[22] P Pansu, Cohomologie $L^{p}$ des variétés à courbure négative, cas du degré 1, from: "Conference on partial differential equations and geometry", Rend. Sem. Mat. Univ. Politec. Torino (1989) 95-120 MR1086210

[23] J Roe, Lectures on coarse geometry, University Lecture Series 31, Amer. Math. Soc. (2003) MR2007488 
[24] K Schmüdgen, Noncommutative real algebraic geometry - some basic concepts and first ideas, from: "Emerging applications of algebraic geometry", (M Putinar, S Sullivant, editors), IMA Vol. Math. Appl. 149, Springer, New York (2009) 325-350 MR2500470

[25] Y Shalom, Rigidity of commensurators and irreducible lattices, Invent. Math. 141 (2000) 1-54 MR1767270

[26] J Špakula, R Willett, Maximal and reduced Roe algebras of coarsely embeddable spaces, J. Reine Angew. Math. 678 (2013) 35-68 MR3056102

[27] Y Stalder, Fixed point properties in the space of marked groups, from: "Limits of graphs in group theory and computer science", (G Arzhantseva, A Valette, editors), EPFL Press, Lausanne (2009) 171-182 MR2562144

[28] K Whyte, Amenability, bi-Lipschitz equivalence, and the von Neumann conjecture, Duke Math. J. 99 (1999) 93-112 MR1700742

[29] $\mathbf{R}$ Willett, G Yu, Higher index theory for certain expanders and Gromov monster groups, I, Adv. Math. 229 (2012) 1380-1416 MR2871145

[30] R Willett, G Yu, Higher index theory for certain expanders and Gromov monster groups, II, Adv. Math. 229 (2012) 1762-1803 MR2871156

[31] R Willett, G Yu, Geometric property (T), Chin. Ann. Math. Ser. B 35 (2014) 761-800 MR3246936

Mathematical Institute, Tohoku University

Sendai 980-8578, Japan

Research Institute for Mathematical Sciences, Kyoto University

Kyoto 606-8502, Japan

School of Science, Tokai University

Hiratsuka 259-1292, Japan

Department of Mathematical Sciences, University of Tokyo

Tokyo 153-0041, Japan

mimura-mas@m.tohoku.ac.jp, narutaka@kurims.kyoto-u.ac.jp,

hiroki.sako@gmail.com, suzukiyu@ms.u-tokyo.ac.jp

http://www.math.tohoku.ac.jp/ mimura/index.html,

http://www.kurims.kyoto-u.ac.jp/ narutaka/,

http://www1.hinocatv.ne.jp/sako-h/research/research.html,

http://www.ms.u-tokyo.ac.jp/ suzukiyu/

Received: 21 May 2014 
